\title{
Machine-Learning-Optimized Aperiodic Superlattice Minimizes Coherent Phonon Heat Conduction
}

\author{
Run $\mathrm{Hu},{ }^{1,2, \dagger}$ Sotaro Iwamoto, ${ }^{2, \dagger}$ Lei Feng, ${ }^{2}$ Shenghong Ju $\odot,{ }^{2}$ Shiqian $\mathrm{Hu},{ }^{2}$ Masato Ohnishi, ${ }^{2}$ \\ Naomi Nagai, ${ }^{3}$ Kazuhiko Hirakawa ${ }^{3,4}$ and Junichiro Shiomi $\odot^{2,5,6, *}$ \\ ${ }^{1}$ State Key Laboratory of Coal Combustion, School of Energy and Power Engineering, Huazhong \\ University of Science and Technology, Wuhan 430074, China \\ ${ }^{2}$ Department of Mechanical Engineering, University of Tokyo, 7-3-1 Hongo, Bunkyo, \\ Tokyo 113-8656, Japan \\ ${ }^{3}$ Institute of Industrial Science, University of Tokyo, Meguro-ku, Tokyo 153-8505, Japan \\ ${ }^{4}$ Institute for Nano Quantum Information Electronics, University of Tokyo, \\ Meguro-ku, Tokyo 153-8505, Japan \\ ${ }^{5}$ Center for Materials Research by Information Integration, National Institute for Materials Science, \\ 1-2-1 Sengen, Tsukuba, Ibaraki 305-0047, Japan \\ ${ }^{6}$ RIKEN Center for Advanced Intelligence Project, 1-4-1 Nihombashi Chuo-ku, 103-0027 Tokyo, Japan
}

(Received 20 June 2019; revised manuscript received 20 March 2020; accepted 14 April 2020; published 4 June 2020)

\begin{abstract}
Lattice heat conduction can be modulated via nanostructure interfaces. Although advances have been made by viewing phonons as particles, the controllability should be enhanced by fully utilizing their wave nature. By considering phonons as coherent waves, herein we design an optimized aperiodic superlattice that minimizes the coherent phonon heat conduction by alternatingly coupling coherent phonon transport calculations and machine learning. The thermal conductivity of the fabricated aperiodic superlattice agrees well with the calculations over a temperature range of 77-300 K, indicating that complex aperiodic wave interference of coherent phonons can be controlled. The thermal conductivity of the aperiodic superlattice is significantly smaller than the conventional periodic superlattice due to enhanced phonon localization. The optimized aperiodic structure is formed by connecting weakly correlated local structures that introduce interference over broad phonon frequencies. Controlling coherent phonons by aperiodic interferences opens a new route for phonon engineering.
\end{abstract}

DOI: 10.1103/PhysRevX.10.021050

\section{INTRODUCTION}

The lattice thermal conductivity, particularly in semiconductors and insulators, has been a key thermophysical property that determines the usability and functionality of materials in various applications such as thermal management, thermal barriers, and thermoelectrics [1]. Over the past few decades, phonon transport in nanostructured materials has drawn increasing attention because introducing interfaces and nanoparticles inside one-, two-, and three-dimensional systems enhances the viability of forming artificial nanostructures [2-6].

\footnotetext{
* To whom correspondence should be addressed. shiomi@photon.t.u-tokyo.ac.jp

${ }^{\dagger}$ R. H. and S. I. have contributed equally to this work.

Published by the American Physical Society under the terms of the Creative Commons Attribution 4.0 International license. Further distribution of this work must maintain attribution to the author(s) and the published article's title, journal citation, and DOI.
}

Subject Areas: Computational Physics, Condensed Matter Physics
Thermal phonons, when scattered by nanostructures, are usually considered as incoherent particles due to their relatively small wavelengths. Nanostructures thus diffusely scatter thermal phonons, which give rise to a reduction in the lattice thermal conductivity. A statistical description is frequently adopted to evaluate the transport of such incoherent particles. Often the Boltzmann transport equation is used to obtain the size-, mode-, and frequencydependent thermal conductivity characteristics [7-9]. In contrast, in this decade, coherent phonon waves have recaptured attention when the experimental lattice thermal conductivities are much lower than the predicted particlebased models in superlattices and nanomeshes by considering the wave-particle duality of phonons [6,10-12]. The mechanism is proposed by resorting to the phonon wave interference effect, which leads to the formation of phononic forbidden band gaps and a reduction in group velocities [13]. Including other approaches such as using the local resonance of phonon waves [14-20], the control of phonon waves, supplementing the advance in the control of phonon particles, should enhance the degree of freedom 
and flexible availability in the manipulation of phonon transport. This will advance the exploration to minimize the lattice thermal conductivity, which is of particular use for thermal barriers and thermoelectrics.

However, experimental observations and manipulations of phonon wave transport by nanostructures (i.e., controlling the coherent effect) are challenging because typical wavelengths of thermal phonons at room temperature are only a few nanometers and phonons lose their phase information (coherence) relatively easily due to scattering. Silicon thin films with periodic $\sim 100$-nm-diameter holes have been a popular system to investigate the coherent effect, but the temperature must be below $10 \mathrm{~K}$ for the effect to influence thermal conductivity [6].

There are two conditions in which the coherent effect is manifested. One is that the interface of the nanostructure needs to be so smooth that phonons preserve the coherence after being scattered at the interfaces. The other is that interface scattering dominates the dephasing mechanism such as inelastic phonon-phonon scattering in the interior. To this end, a suitable platform to experimentally study the phonon coherence effect is superlattices (SLs), which can be synthesized with nanometer layers and coherently bonded interfaces. To date, SLs have been used to reveal various aspects like phonon localization and particle-wave crossover [14-16]. In a study on gallium arsenide (GaAs)/ aluminum arsenide (AlAs) SLs, the measured dependence of thermal conductivity on layer thicknesses agrees with that of the fully coherent phonon transport calculations based on first-principles interatomic force constants, suggesting the manifestation of the coherent effect [14].

Theories on SLs have predicted coherent phonon heat conduction tunability far greater than what has yet to be achieved experimentally by varying the period number and thickness [21-23], interface density [24], interfacial disorder and roughness [25,26], mass mismatch [27], and chemical bonds [27]. The large parameter sensitivity gained by the coherent effect suggests structure optimization is possible. However, the degrees of freedom in such an optimization problem would be enormous. For example, simply considering a SL structure with two kinds of materials $A$ and $B$, and stacking $N$ layers that are either $A$ or $B$ results in as many as $2^{N}$ kinds of SL structures. Searching for a specific structure such as that with the minimum thermal conductivity among the numerous candidates is like looking for a needle in a haystack. Because the enumeration approach is not realistic, an alternative feasible solution is necessary.

Machine-learning-based material informatics (MI) has emerged as the fourth paradigm of material science, following the empirical, theoretical, and computational eras for material development. MI applies the principles of datadriven informatics to materials science to unify the previous three paradigms in an effort to better understand and manipulate the use, selection, manufacturing, qualification, and discovery of materials [28-30]. MI has recently been demonstrated to be useful in various applications with different target physical properties such as steel fatigue prediction [31], stable compound discovery [32], microstructure optimization [33], crystal symmetry to functionality selection [34], and metal-insulator classification [35].

In the field of thermal transport, early works have adopted the concept of high-throughput screening to search for low thermal conductivity compounds from structural databases [36]. More recently, the optimization method has been developed to design nanostructures for a given objective [37]. MI has been proven efficient to identify the optimized structure by calculating only a small percentage of the total candidates. It has been also shown that the method can optimize the balance between multiple properties that are usually in antagonistic relationships (e.g., thermal and electrical conduction in thermoelectrics) [38]. The important finding to date from these theoretical works in the context of coherent phonon heat conduction is that the largest reduction of heat conduction is realized by aperiodic SLs instead of periodic SLs used in most of the experimental studies thus far $[14,15]$.

Therefore, a large and nontrivial step to advance heat conduction control is to experimentally and quantitatively demonstrate superior controllability of coherent phonon heat conduction by aperiodic SLs. In this study, we identify the globally optimal aperiodic structure of GaAs/AlAs SLs from 65536 candidates to minimize thermal conductivity by manipulating coherent phonon transport and taking advantage of MI that couples Bayesian optimization and first-principles-based atomistic Green's function (FP AGF) calculations. The designed optimal structures are subsequently fabricated by molecular beam epitaxy (MBE) and thermal conductivity is measured by time-domain thermoreflectance (TDTR) technique at temperatures ranging from 77 to $300 \mathrm{~K}$. Through these efforts, we demonstrate the ultimate controllability of coherent phonon heat conduction by aperiodic SL, which opens a new route for phonon engineering. Furthermore, the mechanism of the controllability is explained in terms of phonon localization and interference.

\section{METHODOLOGY}

\section{A. First-principles calculation}

As the first step, we obtain the interatomic force constants (IFCs) for the pure GaAs and AlAs crystals. The IFCs are defined as the Taylor expansion coefficients of the force with respect to atomic displacements around equilibrium configurations, as

$$
F_{i, \alpha}=-\Pi_{i, \alpha}-\sum_{j, \beta} \Phi_{i j}^{\alpha \beta} u_{j, \beta}-\frac{1}{2} \sum_{j k, \beta \gamma} \Psi_{i j k}^{\alpha \beta \gamma} u_{j, \beta} u_{k, \gamma},
$$

where $\Phi_{i j}^{\alpha \beta}$ and $\Psi_{i j k}^{\alpha \beta \gamma}$ are second- and third-order IFCs, respectively, the subscripts $(i, j, k)$ are the atom indices, and 
the superscripts $(\alpha, \beta, \gamma)$ are the Cartesian components. The Quantum ESPRESSO package is used to run the density functional theory calculation. Both GaAs and AlAs crystals share the same zinc blende fcc structure with a slight difference in lattice constants: $a=b=c=5.6123 \AA$ for GaAs and $a=b=c=5.633 \AA$ for AlAs. Supercells with $2 \times 2 \times 2,2 \times 2 \times 4$, and $2 \times 2 \times 8$ of the fully relaxed conventional unit cells (UCs) of GaAs and AlAs, which correspond to 64,128 , and 256 atoms, are used. The $4 \times$ $4 \times 4$ Monkhorst-Pack $k$-point mesh is used to sample the electronic states in the first Brillouin zone, and an energy cutoff of 80 Ryd is used for the plane-wave expansion. The Perdew-Zunger local density approximation projector augmented-wave potentials for the $\mathrm{Ga}, \mathrm{Al}$, and As atoms are used to describe the exchange correlation. The corresponding IFCs are obtained by displacing the selected atoms by $\pm 0.01 \AA$ using the ALAMODE package [9]. The forcedisplacement data are fitted according to Eq. (1) with the symmetry properties and translational-rotational invariance conditions by a singular value decomposition algorithm to extract the second- and third-order IFCs for pure crystal atoms. As for the IFCs of the GaAs/AlAs interfacial atoms, we prepare the same supercells as above, but replace the $\mathrm{Ga}$ atoms with $\mathrm{Al}$ atoms in the half of the conventional cells. The supercells consist of 8, 16, and 32 monolayers (MLs), respectively, and are about 1.1, 2.2, and $4.5 \mathrm{~nm}$ long in the $z$ direction. Following the same procedure, IFCs for the interfacial atoms are obtained based on the fully relaxed conventional unit cells. The convergence tests on phonon dispersions and IFCs can be accessed in the Supplemental Material [39].

\section{B. Atomistic Green's function calculation}

In the AGF calculation, a device (or channel) region is sandwiched by two semi-infinite leads. The Green's function $G$ is written as $G(\omega)=\left[\omega^{2} I-H_{d}-\Sigma_{1}-\Sigma_{2}\right]^{-1}$ where $\omega$ is the phonon frequency and $H_{d}$ is the IFC matrix in the device region. $\Sigma_{1}$ and $\Sigma_{2}$ are the contact self-energies, which can be obtained from the surface Green's functions $g_{1}$ and $g_{2}$ as $\Sigma_{1}=\tau_{1} g_{1} \tau_{1}^{\dagger}$ and $\Sigma_{2}=\tau_{2} g_{2} \tau_{2}^{\dagger}$, with $\tau_{1}$ and $\tau_{2}$ being the IFC matrices for the interaction between the atoms in the device region and the two leads. The phonon transmission function across the device is obtained from the Caroli formula as $\Xi(\omega)=\operatorname{Tr}\left(\Gamma_{1} G^{r} \Gamma_{2} G^{a}\right)$, where $\Gamma_{1,2}=$ $i\left[\Sigma_{1,2}^{r}-\Sigma_{1,2}^{a}\right]$ are the level broadening matrices to denote the rate at which phonons enter and exit the leads. $G^{r}$ and $G^{a}$ are the retarded and advanced Green's functions of the scattering region. In the calculations, the periodic boundary conditions are applied in the transverse directions and the Caroli formula can be calculated for full phonon spectrum including short- and long-wavelength phonons. The Landauer formula is used to compute the heat current flowing through the device with two fixed thermostats $T_{L}$ and $T_{R}$ at the two ends, yielding [37]

$$
J=\frac{1}{2 \pi} \int \hbar \omega\left[f_{L}\left(\omega, T_{L}\right)-f_{R}\left(\omega, T_{R}\right)\right] \Xi(\omega) d \omega,
$$

where $f_{L}$ and $f_{R}$ are the Bose-Einstein distributions of phonons. Considering the limit of small temperature difference $T_{L}-T_{R}$ between the two leads, the cross-plane thermal conductance of the device is

$$
G(T)=\frac{\hbar^{2}}{2 \pi k_{B} T^{2} S} \int \omega^{2} \Xi(\omega) \frac{e^{\hbar \omega / k_{B} T}}{\left(e^{\hbar \omega / k_{B} T}-1\right)^{2}} d \omega,
$$

where $T=\left(T_{L}+T_{R}\right) / 2$, and $k_{B}$ and $S$ are the Boltzmann constant and the cross-sectional area, respectively.

The AGF only considers the second-order harmonic interatomic force, and thus, only accounts for the elastic phonon scattering to calculate the coherent thermal conductance. Thermal conductivity, on the other hand, is a property that is defined for diffusive transport through Fourier's law. However, as have been widely done for ballistic heat conduction, we convert the thermal conductance to effective thermal conductivity as $\kappa(T)=G(T) \times L$, where $L$ is the total length of the superlattice. This allows us to compare the thermal conductivity directly with the thermal conductivity measured in the experiments, which is also an effective one. Note that the effective thermal conductivity here can vary with the size (classical size effect) because the transport is ballistic, but that is the same in the measurements. The convergence of thermal conductance on the supercell size is checked by performing the AGF calculations for $2 \times 2 \times 2,2 \times 2 \times 4$, and $2 \times 2 \times 8$ supercells with one GaAs/AlAs interface, and the corresponding values of the thermal conductance are 187.69, 188.35 , and $185.65 \mathrm{MW} /\left(\mathrm{m}^{2} \mathrm{~K}\right)$, respectively. This is consistent with the size convergence of IFCs described in the Supplemental Material [39].

\section{Machine-learning-based optimization}

As shown in Fig. 1, we first prepare the candidate pool with all possible structures by labeling the GaAs unit layer (UL) as 0 and the AlAs UL as 1 . If the total number of the SL UL is $N$, there will be $2^{N}$ kinds of candidate structures in the pool. Here, we discretized the GaAs/AlAs superlattice film with a fixed thickness of 16 ULs, where each unit is either GaAs or AlAs, to express the structural variations of the SLs. This gives rise to $2^{16}(=65536)$ possible candidate structures. The SL structure is sandwiched by two semi-infinite GaAs leads to allow thermal transport. For different UL sizes, the corresponding IFCs are obtained by the first-principles calculation in advance. Then we randomly select $m$ candidates as the initial SL structures and calculate the corresponding thermal conductivities. For each candidate structure, we construct the dynamical matrix for the 16-UL SL structure by calling for the corresponding IFCs and mass matrices. Then by 


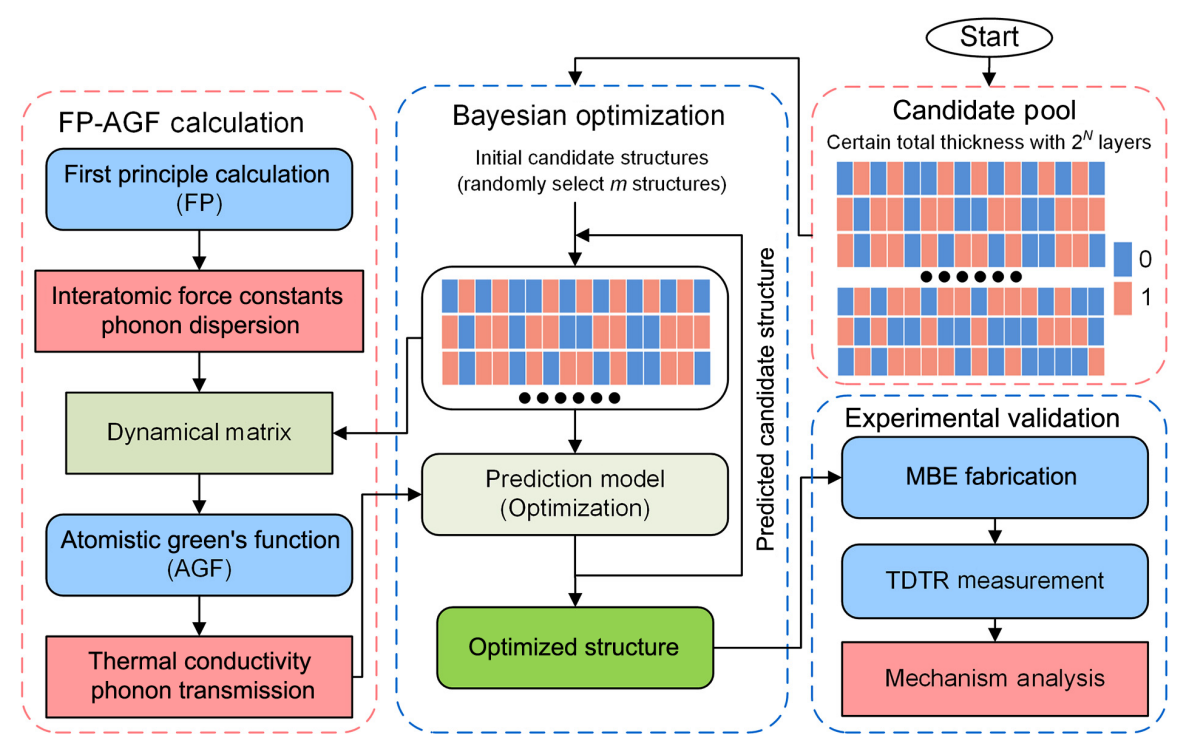

FIG. 1. Flowchart of the optimal control for coherent phonon heat conduction. Structural optimization for minimum thermal conductivity is achieved by alternately coupling Bayesian optimization and first-principles (FP) atomistic Green's function (AGF) calculation, fabrication by molecular beam epitaxy (MBE), and measurements by the time-domain thermoreflectance (TDTR) method.

substituting the dynamical matrix into the FP AGF calculation, the corresponding thermal conductivity of each 16-UL SL candidate structure is obtained.

As for the MI implementation, we employ the opensource Bayesian optimization library СОмВо to perform the machine-learning-based optimization process [40]. Taking the $m$ initial structures as the original training data set, we employ Thompson sampling to predict the next $n$ candidate structures from the candidate pool according to the probability of being optimal. The prediction model used is the Bayesian linear regression model as $y=\mathbf{w}^{T} \chi(\mathbf{x})+\varsigma$, where $\mathbf{x}$ is $N$-dimensional vector corresponding to a candidate structure, $\chi: \mathfrak{R}^{N} \rightarrow \mathfrak{R}^{\ell}$ is a feature map, $\mathbf{w} \in$ $\Re^{\ell}$ is a weight vector, and $\varsigma$ is the noise with normal distribution $N\left(0, \sigma^{2}\right)$. How to randomly select $m$ initial candidates is also important and the rule here is to make sure the inner product $\chi(\mathbf{x})^{T} \chi\left(\mathbf{x}^{\prime}\right)$ approximates the Gaussian kernel of width $\eta$, i.e., $\exp \left[-\left\|\mathbf{x}-\mathbf{x}^{\prime}\right\|^{2} /\left(2 \eta^{2}\right)\right]$. The performance of сомво is determined by the hyperparameters $\sigma$ and $\eta$. Before optimization, the hyperparameters are initialized according to a heuristic procedure. At every round of Bayesian optimization, $n$ candidates are added to update the original basic training data set. By maximizing the type-II likelihood with ADAM algorithm, we can obtain the updated hyperparameters at every round to accelerate the search for the optimal one. More details on the сомво optimization can be found elsewhere $[37,38,40]$.

\section{Sample fabrication}

The samples used in this study are grown on (100)oriented semi-insulating GaAs substrates by MBE. The reference sample is prepared by growing three sets of AlAs/ GaAs alternating layers (4.5/4.49/4.5/4.49/4.5/4.49/ 4.5/4.49/4.5/4.49/4.5/4.49/4.5/4.49/4.5/4.49) (bold: AlAs, plain: GaAs, unit: nm) successively on a $100-\mathrm{nm}-$ thick GaAs buffer layer. The surface is capped by 5-nmthick GaAs layer to avoid oxidation of the active region. The optimized sample is prepared by growing three sets of AlAs/GaAs alternating layers (4.5/4.49/9/4.49/9/4.49/ 4.5/4.49/4.5/4.49/4.5/8.98/4.5) (bold: AlAs, plain: GaAs, unit: $\mathrm{nm}$ ) successively on a 100-nm-thick GaAs buffer layer. Similarly, the surface is capped by $5 \mathrm{~nm}$-thick GaAs layer.

\section{E. Time-domain thermoreflectance measurements}

The thermal conductivity of the SLs is measured using the TDTR technique [41]. TDTR is an optical pump and probe technique using Ti:sapphire femtosecond pulsed laser (reputation rate $80.21 \mathrm{MHz}$, pulse width $200 \mathrm{fs}$, the wavelength is $800 \mathrm{~nm}$ ). The laser is split into a pump beam and a probe beam by a beam splitter. The pump beam, whose wavelength is changed to $400 \mathrm{~nm}$ by a BIBO, induces a pulsed temperature change on the sample surface, after being modulated with electro-optical modulator at a frequency of $11.05 \mathrm{MHz}$. On the other hand, the probe beam is reflected on the sample surface after being delayed by mechanical stage, and the variation of the reflected power is translated to the temperature change on the sample surface through thermoreflectance. A lock-in amplifier picks out the signal from reflected probe beam at the modulation frequency, which consists of in-phase voltage $\left(V_{\text {in }}\right)$ and out-of-phase voltage $\left(V_{\text {out }}\right)$. Thermal conductivity and interface thermal conductance are obtained by fitting the solution of a theoretical physical model to the measured ratio $-V_{\text {in }} / V_{\text {out }}$. Details of the analysis are described in previous publications [14,41]. 
The $1 / e^{2}$ diameter of the pump and the probe was fixed to 23.9 and $11.5 \mu \mathrm{m}$, respectively. Prior to measurement, aluminum film ( $\sim 80 \mathrm{~nm}$ thick) was deposited on the sample as a transducer for the TDTR measurement. The thickness of the aluminum film was determined by simultaneously performing the aluminum deposition on a glass substrate with known thermal conductivity. Thermal conductivity here includes the interfacial thermal conductance (resistance) between the SL and the GaAs substrate, which is consistent with the calculation, noting that the interface is the same as the ones inside the SL.

\section{RESULTS AND DISCUSSIONS}

\section{A. MI optimization}

The optimization process, as shown in Fig. 1, begins by randomly selecting 20 candidates and calculating their thermal conductivity. The 20 pairs of descriptors and thermal conductivity values serve as the initial training data for a Bayesian regression function to estimate the predictive distribution of thermal conductivity for all the remaining candidates [40]. This provides 20 candidates that are predicted to have small thermal conductivities. Next, their thermal conductivity values are calculated and added to the training data together with their descriptors. By repeating this procedure, the calculation of thermal conductivity is scheduled optimally, and the optimized structure can be found quickly. Previous works have shown that repeating this for only a small percentage of the entire population of candidates is sufficient to find the optimal structure [37-40]. In this work, we calculate 1760 structures, merely $2.7 \%$ of the total possible candidates to achieve the global optimal structure (more details on the optimization are available in the Supplemental Material [39]).

Performing the optimization with a two-UC-thick UL gives the GaAs/AlAs SL structure with the smallest thermal conductivity of 1001010101101101 downwardly. Figure 2(a) shows this using the descriptors defined above as labels. The optimal GaAs/AlAs SL is an aperiodic structure, as previously found numerically for a silicon-germanium SL [37]. For additional comparisons between the calculated and measured SLs, we expanded the UL four times to eight (a)

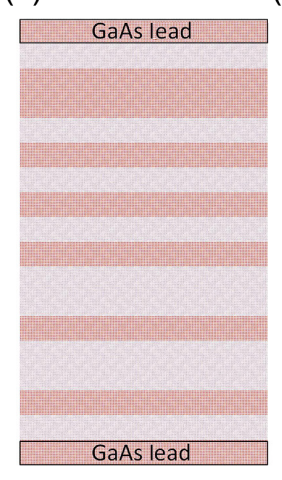

(b)

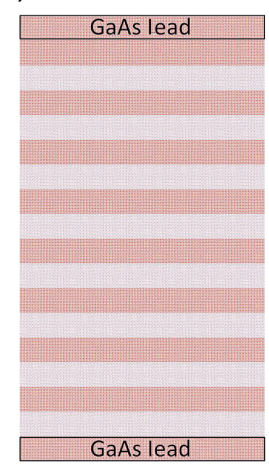

(c)

(e)
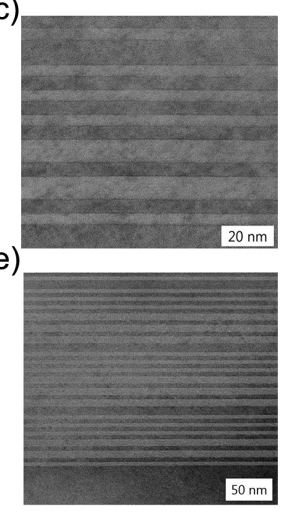

(d)

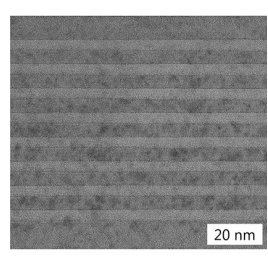

(f)

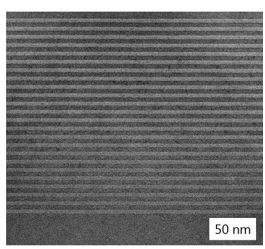

(g)

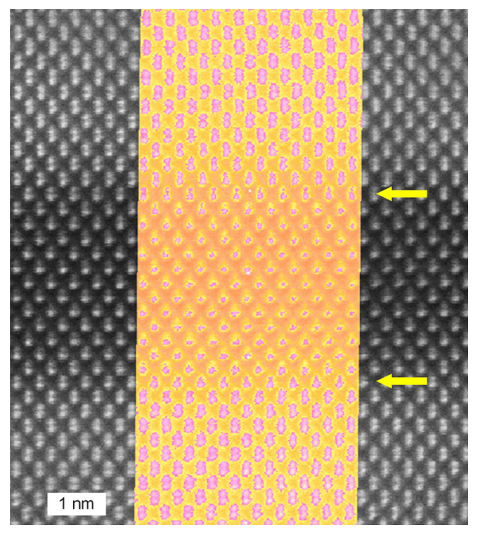

GaAs

$\mathrm{Al} / \mathrm{Ga}$

transitional brightness

AlAs

$\mathrm{Al} / \mathrm{Ga}$

transitional brightness

GaAs

FIG. 2. Designed and fabricated SL samples. (a) Schematics of the global optimal GaAs/AlAs SL structure obtained by materials informatics (MI) from the candidate pool of $2^{16}(=65536)$ structures. Red and gray layers denote GaAs (0) and AlAs (1), respectively. Binary label is 1001010101101101 from the top to bottom with GaAs leads on the two ends. (b) Schematic of the reference periodic structure with a binary label of 0101010101010101 . TEM images of the fabricated (c) optimal aperiodic and (d) reference periodic GaAs/AlAs SL structures, corresponding to the schematics in (a) and (b), respectively. To magnify the thermal conductivity difference between the optimal aperiodic and reference periodic structures, each structure is repeated for three times to obtain longer GaAs/AlAs SL structures, as shown in (e) and (f), respectively. (g) Atomistic-resolution TEM to identify the monolayer interfacial roughness denoted by the arrows. 
UCs $(4.6 \mathrm{~nm})$ to ensure that the UL thickness becomes sufficiently larger than the monoatomic-layer interfacial roughness, which inevitably appears in the fabricated SLs. This is justified by the finding that the order of thermal conductivity values among the candidates remains the same as the UL thickness is changed from two to eight UCs (more details on the convergence tests can be found in the Supplemental Material [39]). Such self-similarity supports that the optimal structure is also 1001010101101101 when the UL is eight-UCs thick. The performance of the optimized structure is demonstrated by comparing it with the finestperiodic SL with the same thickness, which consists of alternating GaAs and AlAs ULs. This gives the largest number of interfaces [Fig. 2(b)]. The optimized aperiodic SL exhibits a significantly smaller thermal conductivity throughout the temperature range [Fig. 3(a), solid lines]. Since a periodic SL has more GaAs/AlAs interfaces, the superiority of an aperiodic SL suggests successful engineering of coherent phonon heat conduction.

(a)

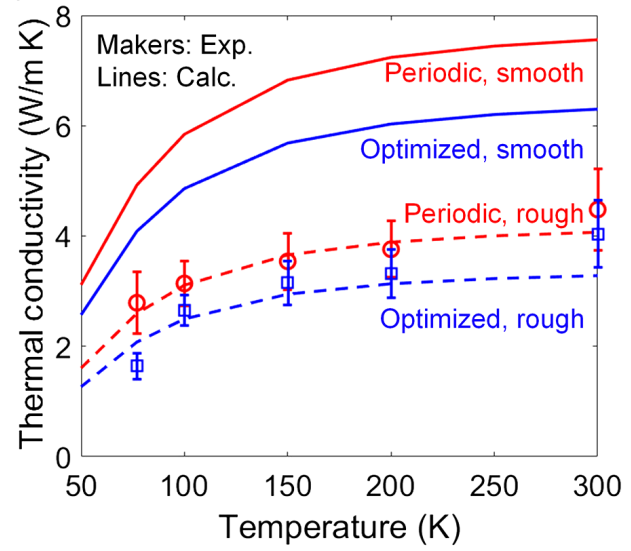

(c)

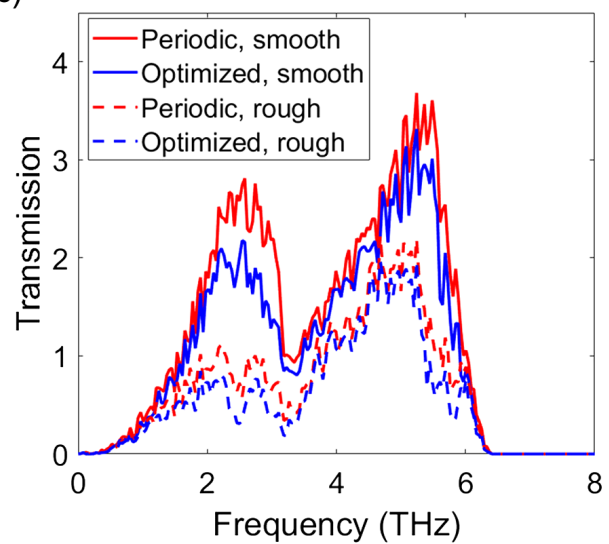

\section{B. Experimental realization}

The focus of this work is to experimentally demonstrate the minimization of the coherent phonon heat conduction. To this end, we fabricate aperiodic and periodic GaAs/AlAs SLs on a GaAs substrate using MBE. The transmission electron microscope (TEM) images of the 16-UL optimized-aperiodic and periodic structures are shown in Figs. 2(c) and 2(d), where the total thicknesses are 76.9 and $74.5 \mathrm{~nm}$, respectively. Both the GaAs and AlAs UL are about $4.5-\mathrm{nm}$ thick. For a clearer demonstration of the difference in thermal conductivity between the optimized aperiodic SL and its periodic counterpart, we also fabricated thicker samples by repeating the 16-UL structures for three times. Figures 2(e) and 2(f) show the TEM images of the resulting 48-UL structures. The actual total lengths of the SL samples are 203.3 and $218.0 \mathrm{~nm}$, respectively. To further identify the atomic interface roughness, we perform an atomistic-resolution TEM measurement as shown in Fig. 2(g). For clarity, the contrast is magnified in the strip

(b)



(d)

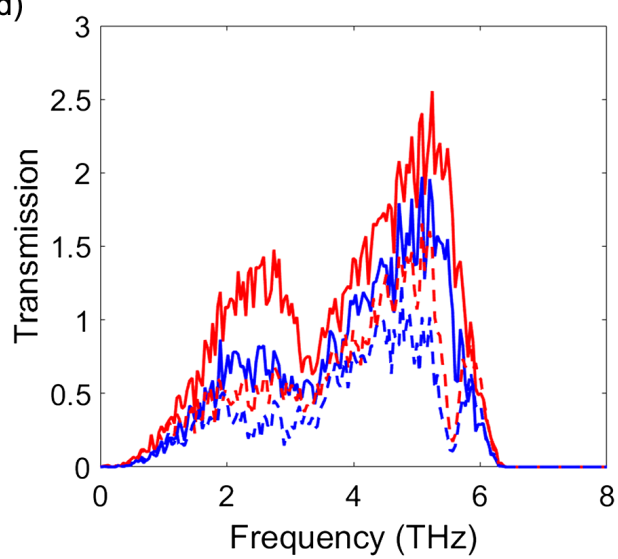

FIG. 3. Thermal conductivity and phonon transmission characterization of 16-UL and 48-UL SL structures. Experimental and calculated thermal conductivities of (a) the 16-UL and (b) 48-UL optimized aperiodic and periodic SL structures for a temperature range from 50 to $300 \mathrm{~K}$. Calculated thermal conductivity of the optimized aperiodic SLs (blue) is always smaller than that of the periodic SLs (red) throughout the temperature range. Calculated thermal conductivity for rough interfaces (dashed line) is smaller than that for the smooth interfaces (solid line) and is consistent with the experimental measurements (red circles for periodic SLs, blue squares for optimized aperiodic SLs). Green dotted line in (b) denotes the calculated thermal conductivity of the exact structure of the fabricated 48UL-SL obtained by TEM. Corresponding phonon transmission functions for the (c) 16-UL and (d) 48-UL structures. 
region in the middle of the image. A sharp change in the brightness can be seen at the GaAs/AlAs interface due to of the brightness difference between the Ga sites and $\mathrm{Al}$ sites with a monolayer in between exhibiting the intermediate brightness denoted by the arrows. Such measurement confirms that the fabricated superlattice has nearly perfect interface with monolayer roughness. The thicknesses of the fabricated samples slightly deviate from the design due to the systematic error in the MBE deposition rate. Even with this deviation, the superiority of the optimized structure is evident, and it can be incorporated in thermal conductivity calculations when comparing with experiments.

Thermal conductivity of the fabricated SL is measured using the TDTR technique over a temperature range from 77 to 300 K. Figures 3(a) and 3(b) show the measured and calculated values of thermal conductivity for 16-UL and 48-UL samples, respectively. Note that the thermal conductivity of the 48-UL sample is higher than that of the 16-UL sample due to the size effect of the ballistic heat conduction induced when the total thickness is smaller than the phonon mean-free-path [14]. For both 16-UL and 48-UL samples, the thermal conductivity of the optimized aperiodic samples is always lower than that of the periodic samples.

To compare the calculations with experiments, we incorporate the monolayer roughness at the interfaces in the calculations by adopting a model of interdiffusion between $\mathrm{Ga}$ and $\mathrm{Al}$ atoms. This is done by exchanging $50 \%$ of $\mathrm{Ga}$ and $\mathrm{Al}$ atoms in the atomic layers closest to the interface. Among the unique combinations of choosing four $\mathrm{Ga}(\mathrm{Al})$ atoms from eight $\mathrm{Ga}(\mathrm{Al})$ atoms in the interface monolayers of $2 \times 2 \times 2$ conventional UCs system, following the strategy in the previous study [14], we calculate the thermal conductivity of nine cases and averaged them to obtain the value with rough interface.

As for the 48-UL structure, the total lengths of the SL samples are 203.3 and $218.0 \mathrm{~nm}$ for the optimized aperiodic and the periodic structures, respectively. It is seen that there are missing atom layers in the optimized aperiodic 48-UL structure. By the TEM observation, we find that the fabricated aperiodic 48-UL structure is one atom layer short in the thick AlAs layers (digits of " 11 "). Therefore, we recalculate the thermal conductivity for the actual structure. As shown in Fig. 3(b) (denoted by optimized, rough, exact structures), the calculated thermal conductivity of the actual structure agrees well with that of experiment.

As in Figs. 3(a) and 3(b), by considering the interfacial roughness, the thermal conductivities of the experiments and calculations agree well for both the optimal aperiodic and the finest periodic SLs. The thermal conductivity slightly drops as temperature increases from $200 \mathrm{~K}$, which may be a sign that the inelastic phonon-phonon scattering gradually becomes more important around $300 \mathrm{~K}$. The overall agreement validates the AGF calculations with the
IFCs based on first principles and more importantly suggests that coherent phonon heat conduction is successfully manipulated in the fabricated SL samples. To understand the superiority of the optimized structure from a spectral viewpoint, Figs. 3(c) and 3(d) plot the phonon transmission functions of the optimized aperiodic and periodic structures, respectively. Overall, in both the 16-UL and 48-UL cases, the phonon transmissions of the optimized aperiodic structures are lower than those of the periodic structures in the entire frequency range, except for the lowest frequency range, where the longwavelength phonons tunnel through the entire structure. The cutoff frequency of the transmission function (6.37 THz) corresponds to the highest overlapping frequency of the bulk phonon density of states (DOS) of GaAs and AlAs single crystals. The frequency range of optical phonons in GaAs corresponds to the band gap of AlAs (Supplemental Material, Fig. S1 [39]). Consequently, there is no overlap for the optical phonons. Therefore, the two peaks in the transmission function in Figs. 3(c) and 3(d) correspond to the longitudinal and transverse acoustic modes. The suppressed transmission function over a broad frequency range suggests that thermal conductivity in the optimized aperiodic structure is minimized not by engineering specific frequency bands but by more complex interference events involving a range of coherent phonons.

\section{Phonon localization}

To look into the mechanism, we perform phononlocalization analysis. We calculate the localized density of states (LDOS) from Green's function as $\rho\left(\mathbf{r}_{i}, \omega\right)=$ $-(1 / \pi) \operatorname{Im~G}_{i i}(\omega)$ and the DOS as $\rho(\omega)=N^{-1} \sum_{i} \rho\left(\mathbf{r}_{i}, \omega\right)$ with subscript $i$ standing for the atom site. Then the frequency-dependent generalized inverse participation ratio (IPR) can be obtained as $I(\omega)=\sum_{i} \rho\left(\mathbf{r}_{i}, \omega\right)^{2} /$ $\left[\sum_{i} \rho\left(\mathbf{r}_{i}, \omega\right)\right]^{2}$, which provides a quantitative measure of phonon localization [42,43]. An IPR value of 1 indicates that phonons are totally localized, whereas the delocalized phonons have an IPR value $\sim 1 / N_{m}$, where $N_{m}$ is the total number of atoms in the system. Figure 4(a) shows the calculated IPR of both the optimized aperiodic and the finest periodic SLs, where the yellow dashed lines denote the IPR of a pure GaAs crystal. The results indicate that the optimized aperiodic structure has a stronger localization than the periodic one. The localization is strongest in the optical phonon frequency range above $6.37 \mathrm{THz}$. However, as discussed above, this does not contribute to the difference in the elastic heat conduction between the optimal aperiodic and finest periodic structures.

Looking at the IPR below $6.37 \mathrm{THz}$ [Fig. 4(b)], the localization of the acoustic phonon is stronger in the optimized aperiodic structure than that in the finest periodic structure. Since the IPR only quantifies the magnitude of phonon localization per mode, to relate it to the impact on thermal conductivity, the number of modes must be 
(a)

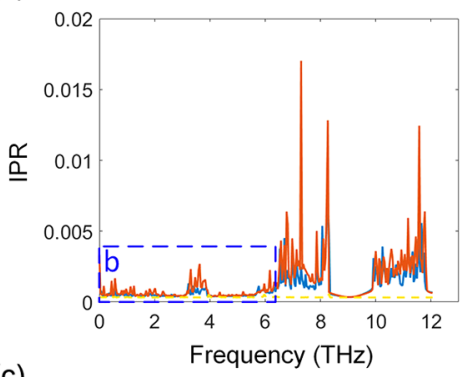

(c)

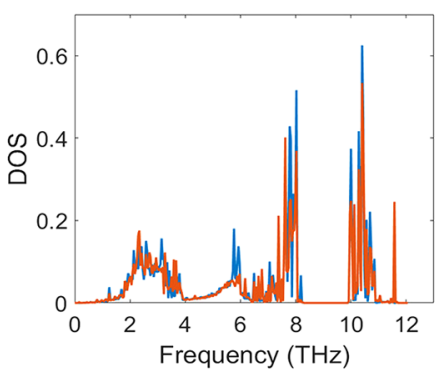

(b)

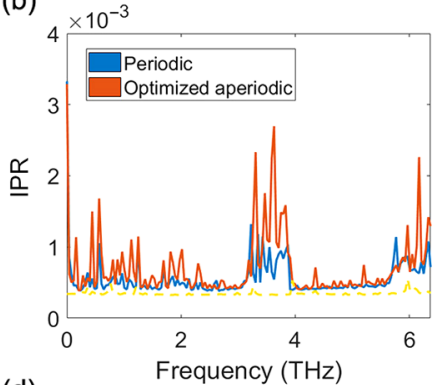

(d)

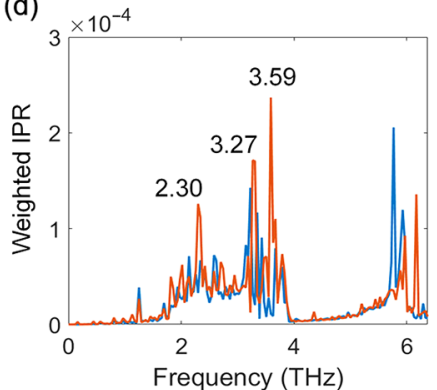

(e)

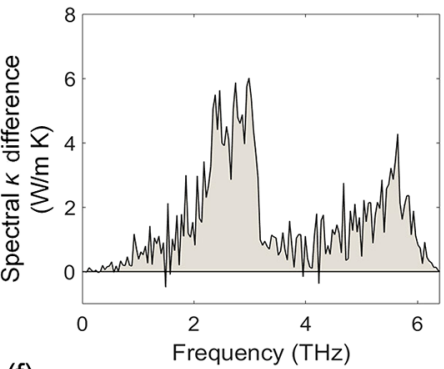

(f)

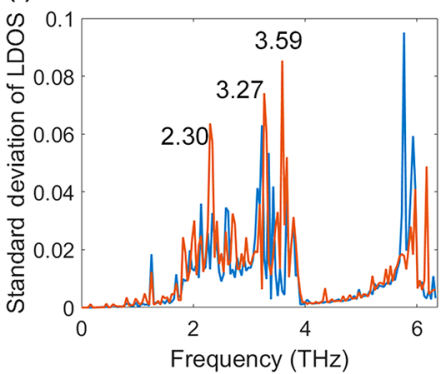

(g)

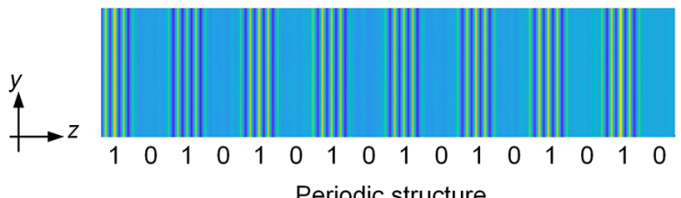

(h)
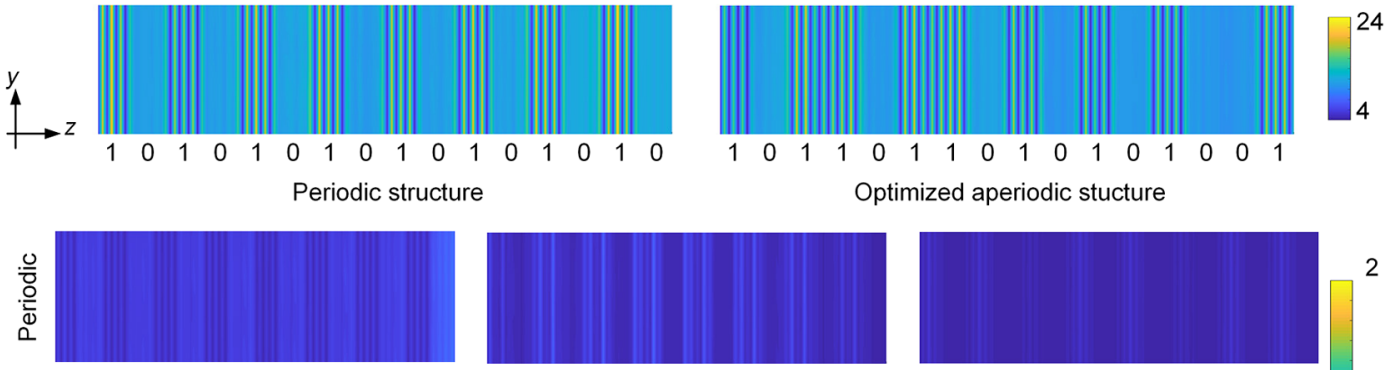

Optimized aperiodic stucture

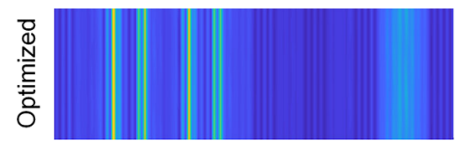

@2.30 THz



@3.27 THz



@3.59 THz

(i)

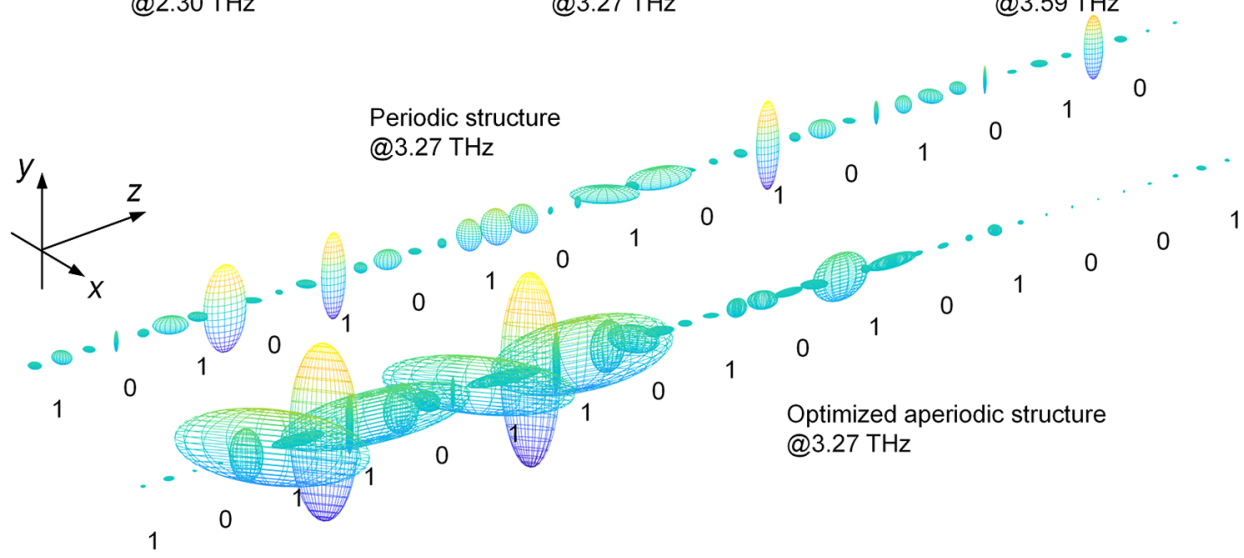

FIG. 4. Phonon-localization analyses of the periodic and the optimized aperiodic structures. Comparison of the frequency-dependent inverse participation ratios (IPR) $I(\omega)$ of the two structures in the (a) full and (b) acoustic phonon frequency ranges. Blue-dashed square in (a) denotes the acoustic phonon range and is magnified in (b). Yellow-dashed lines denote the IPR for pure GaAs crystals with a small and uniform IPR. (c) Spectral DOS of the two structures in the full phonon frequency range. (d) Spectral weighted IPR in the acoustic phonon frequency range. (e) Spectral thermal conductivity difference between that of the periodic structure and that of the optimized aperiodic structure. (f) Spectral standard deviation of LDOS of the two structures in the acoustic phonon frequency range. (g) Projected LDOS distribution on the $y-z$ plane with only the acoustic phonons in the periodic and the optimized aperiodic structures. (h) Projected LDOS in the $y-z$ plane of the two structures at 2.30,3.27, and 3.59 THz. (i) Strength of the phonon localization along the thickness direction ( $z$ axis) of the two structures at $3.27 \mathrm{THz}$. Length of the ellipsoids denotes the averaged LDOS in the $x, y$, and $z$ directions in each monolayer. 
considered. Thus, we defined the weighted IPR as $I_{W}(\omega)=$ $\operatorname{DOS}(\omega) \times I(\omega)=(1 / N)\left[\sum_{i} \rho\left(\mathbf{r}_{i}, \omega\right)^{2} / \sum_{i} \rho\left(\mathbf{r}_{i}, \omega\right)\right]$. The spectral DOS and $I_{W}$ are shown in Figs. 4(c) and 4(d), respectively. Except for the peaks of the periodic structure around $5.8 \mathrm{THz}$, there are distinctly more and stronger peaks in the $I_{W}$ of the optimized aperiodic structure. This indicates that the localization effect of acoustic phonons is more significant. The $I_{W}$ profile of the optimized aperiodic structure is similar to the difference between the spectral thermal conductivities of the optimized aperiodic and periodic structures plotted in Fig. 4(e), confirming that the enhanced localization of acoustic phonons by the aperiodicity is the primary cause of the reduced thermal conductivity.

To gain a further understanding of the localized mode, we associate the above results to LDOS. Note that the weighted IPR is related to the magnitude of local variations in LDOS. Hence, when calculating the standard deviation (SD, $\left.\Delta \rho(\omega)=\sqrt{\left\langle\rho(\omega)^{2}\right\rangle_{i}-\langle\rho(\omega)\rangle_{i}^{2}}\right)$ of LDOS at each frequency, as shown in Fig. 4(f), a clear correlation can be observed through the spectral thermal conductivity difference between the periodic and the optimized aperiodic structures in Fig. 4(e). Then the total thermal conductivity difference can be obtained by integrating the spectral thermal conductivity, i.e., the shadow area, over frequency. Note that the LDOS distribution can be efficiently computed without an explicit diagonalization of the Hamiltonian and is robust even in the presence of interactions to describe the localization properties [44]. The $x$-direction-accumulated LDOS projection of only the acoustic phonons in the $y-z$ plane of the periodic and the optimized aperiodic structures are shown in Fig. 4(g). The structure configurations can clearly be observed. Phonons are localized in the AlAs layers, more specifically, the As atoms in the AlAs layers, while the phonons in the GaAs layers are uniformly distributed. The distinct LDOS difference in As and Al atoms leads to the phonon localization at the As atom in the AlAs layer. This can be understood as the masses of the $\mathrm{Ga}$ and $\mathrm{As}$ atoms are similar while that of the $\mathrm{Al}$ atom is almost three times smaller, inhibiting the kinetic energy transfer between the monolayers of $\mathrm{Al}$ and As atoms.

For the three typical peak frequencies of weighted IPR in Fig. 4(d), the corresponding LDOS projections are shown in Fig. 4(h). For each frequency, the phonons are localized at different places in the optimized structure, while all the phonons are delocalized in the periodic structure. For the $3 \mathrm{D}$ visualization of phonon localization, we extract the LDOS at $3.27 \mathrm{THz}$ and draw ellipsoids, whose three axes are determined by the LDOS of the $x, y$, and $z$ components along the SL thickness direction. The dimensions of the ellipsoids are relatively uniform for the periodic structure, but are vastly different for the optimized aperiodic structure, indicating the occurrence of localization of coherent thermal phonons [Fig. 4(i)].
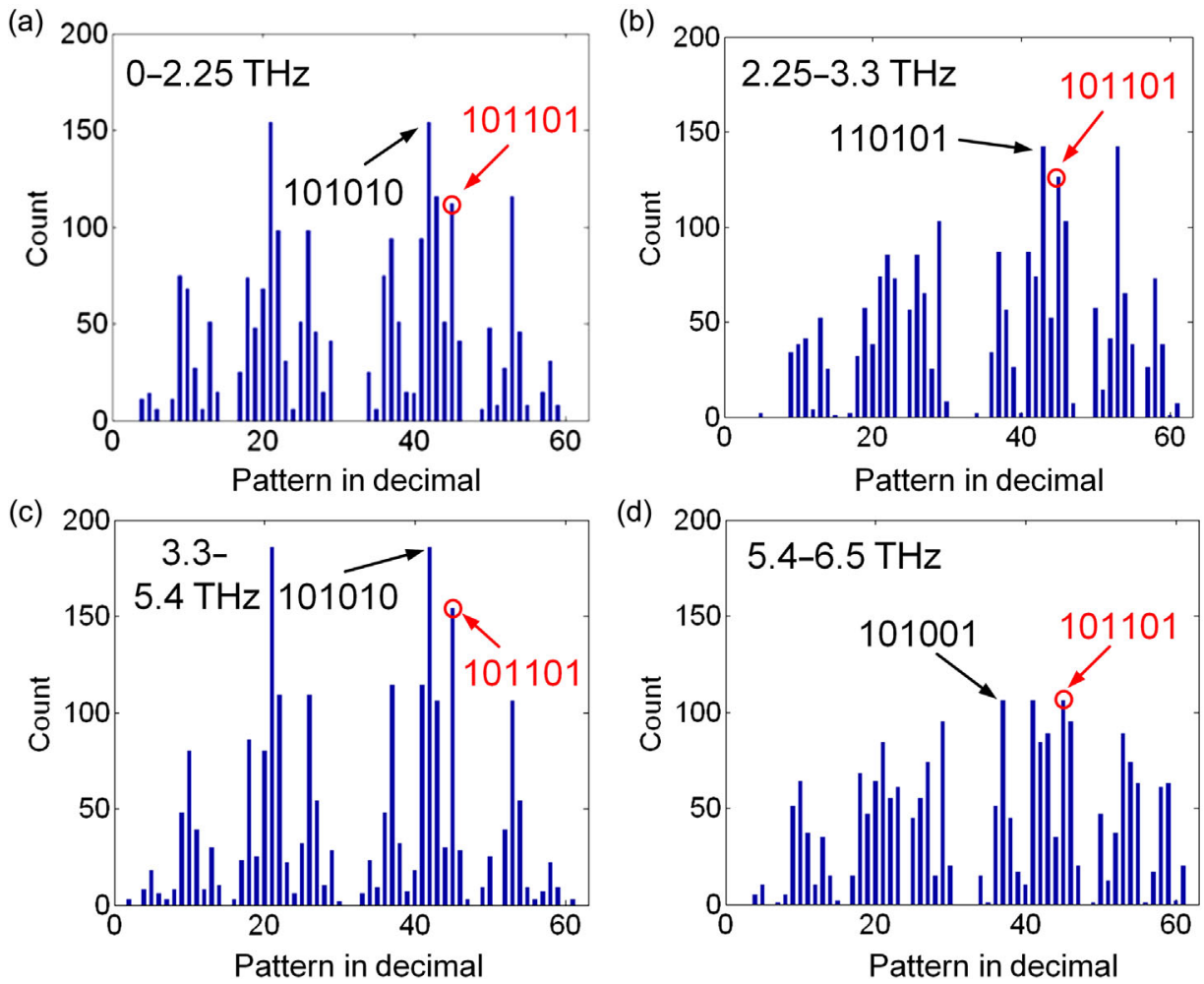

FIG. 5. Histograms of local 6-UL patterns of 100 structures that give small thermal conductivity in the specific four frequency ranges. Four key local structures are identified: 101010, 110101, 101001, and 101101, which are present in the optimized SL. 
(a) Optimized with overlapping Length $=16 \mathrm{ULS}$
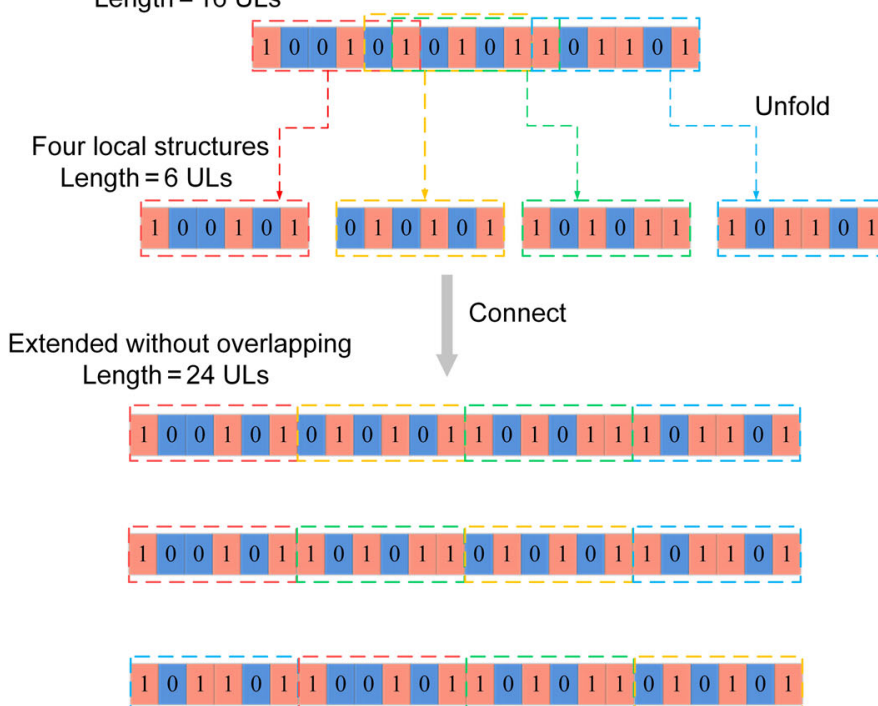

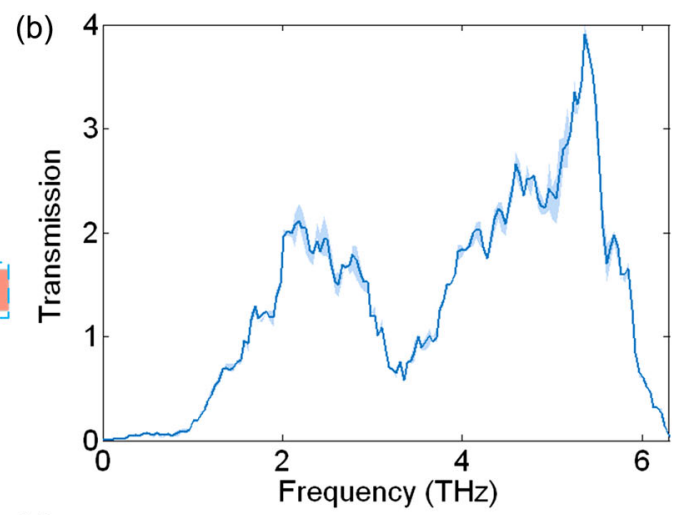

(c)

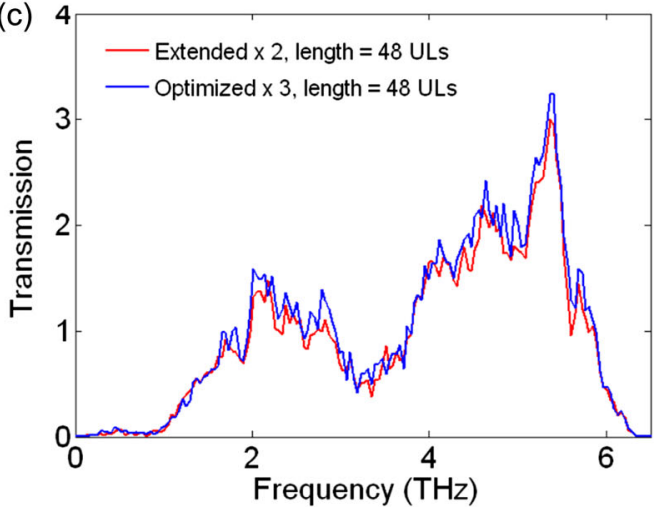

FIG. 6. Local structure independence and correlation examination. (a) Schematic for the optimized SL (16-ULs) and the extended SL (24-ULs). (b) Averaged phonon transmission of the 24 extended SLs, i.e., all possible sequences when serially connecting the four key local structures 101010, 110101, 101001, and 101101. The shadowed region denotes the standard deviation. (c) Comparison of phonon transmissions of optimized SL and the extended SL (averaged) made to the same lengths (48ULs) by repeating three times and twice, respectively.

\section{Local pattern analysis}

The above localization analysis suggest the mechanism to reside in the local structures, which should give rise to phonon interference effects. Therefore, we perform phonon pattern analysis to statistically identify the local structural patterns that are important to reduce phonon transmission and thermal conductivity in four different frequency ranges from low to high frequency as shown in Supplemental Material, Fig. S16 [39]. The statistical histograms of 6-UL patterns are shown in Fig. 5, in which the $x$-axis coordinate denotes the pattern in decimal. For instance, the $x$-axis coordinate of 20 means 010100 with six digits. Clearly, the histograms are different in each frequency range, implying that the local patterns that inhibit phonon transport depend on the frequency range. The local structures that appear most frequently in small thermal-conductivity structures for the four frequency ranges are 101010, 110101, 101010, and 101001, respectively. Furthermore, 101101 also appears as the top local structures in all the frequency ranges, thus the top four local structures are 101010, 110101, 101001, and 101101. Since the local structures tend to give the minimum thermal conductivity at each frequency range, the more local structures an SL has, the smaller its overall thermal conductivity will be. The optimized aperiodic SL has all these local structures, as shown in Fig. 6(a), which is the reason for its minimum thermal conductivity.

To further understand the function of the key local structures in the optimized SL, we unfold the optimized SL to 101010, 110101, 101001, and 101101 and connect them in serial (hereafter, extended SL) as illustrated in Fig. 6(a) to compare the performance with the optimized SL. This is to check if the effect of local structures can be considered separately. Before comparing with the optimized SL, to check if 101010, 110101, 101001, and 101101 are separable (weakly correlated) in terms of phonon transmission, we check the influence of the sequence of four local structures in the extended SL on the phonon transmission. We calculate the phonon transmissions for all the ${ }_{4} P_{4}=24$ possibilities [Fig. 6(a)], and the obtained average profile and the standard deviation are plotted as the blue curve and shadowed region in Fig. 6(b), respectively. As seen in the small standard deviation, the dependence of the phonon transmission on the sequence is weak, suggesting the weak correlation between the local structures. Now to compare the extended SL and optimized SL, the problem is that their lengths are different; the extended SL is 24-UL long and the optimized SL is 16-UL long, which prevents comparison for ballistic transport systems. Therefore, we made the 
(a)

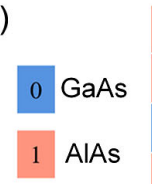

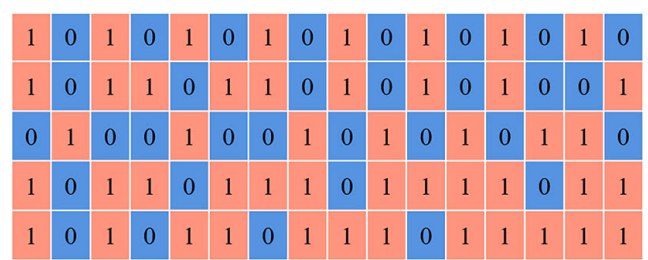

Periodic

Optimized aperiodic

Reversed

Graded

Fibonacci (b)

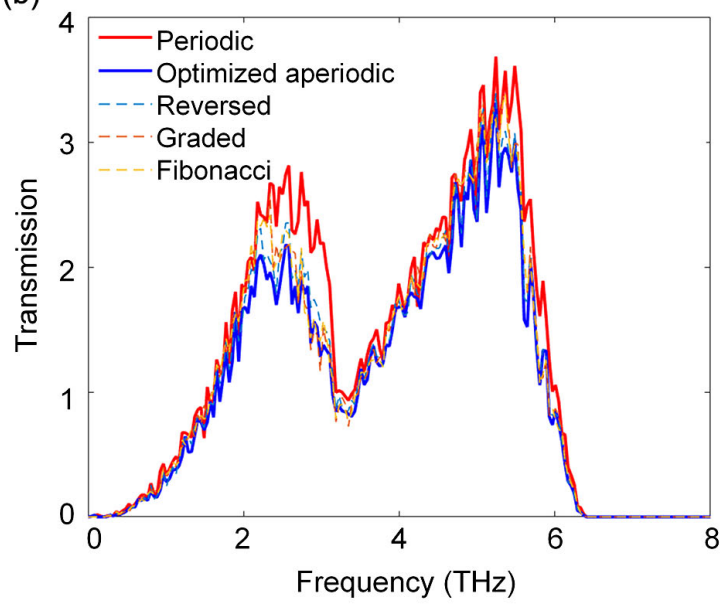

(c)

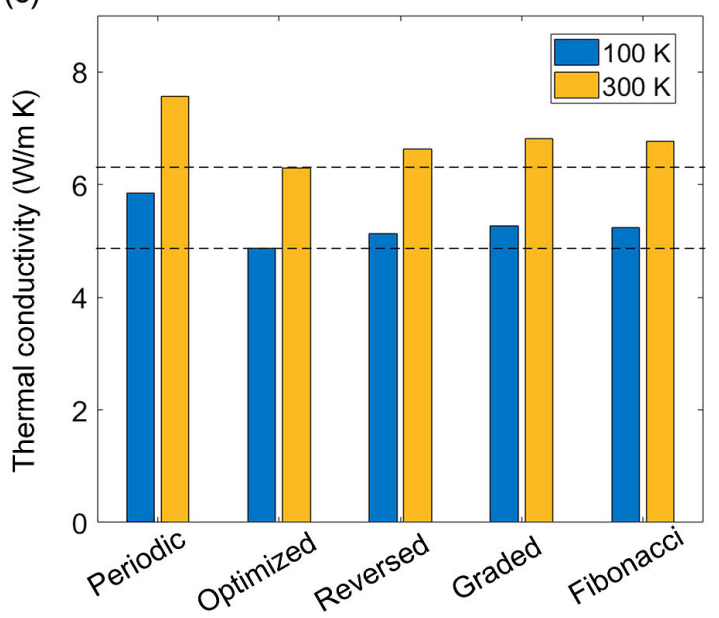

FIG. 7. Comparison of the current optimized structures and other representative aperiodic structures: reversed, graded, and Fibonacci structures. (a) Schematic of the SL configuration for the different structures. (b) Phonon transmission spectra and (c) thermal conductance of the periodic, optimized, reversed, graded, and the Fibonacci structures. Black dashed lines in (c) are the guide to eye.

comparison for 48-UL systems by repeating the extended SL twice and the optimized SL three times. In Fig. 6(c), the blue and red curves denote the phonon transmission of optimized and extended phonon transmissions, and the difference is small. From Fig. 6, we may conclude that the local structures are nearly independent and weakly correlated in inhibiting the phonon transport and thus thermal conductivity, and the current optimized aperiodic structure is formed by connecting various local structure with and without overlaps that introduce interference over broad phonon frequencies simultaneously.

With Figs. 4-6, we could find that the optimized aperiodic structure can minimize thermal conductivity because different parts of the structure separately localize phonons at different frequency ranges, resulting in inhibition of thermal transport in the entire spectrum. It is important here to state that the localization is not Anderson localization, and thus, it is not the randomness of the structure but the specifically designed aperiodic structure that matters on reducing the total thermal conductivity. This is checked by statistically analyzing the distribution of phonon transmission histogram in Supplemental Material, Fig. S17 [39], and confirming that the selected structures with small thermal conductance and randomly selected ones show no difference in the goodness of fitting to the lognormal distribution, which is an indicator of Anderson localization [45]. Details of the analysis can be found in the Supplemental Material [39].

\section{E. Other aperiodic structures}

Further, we compare the phonon transmission with other typical aperiodic structures, including the reversed, graded, and Fibonacci structures. As denoted by Fig. 7(a), the reversed structure is produced by exchanging 0 (GaAs layer) and 1 (AlAs layer) in the optimized aperiodic structure. The graded structure is produced by gradually increasing the number of consecutive 1 as $1,2,3$, and 4 . The Fibonacci structure is realized by changing the number of consecutive 1 following the Fibonacci sequence as 1,1 , 2,3 , and 5 .

Figure 7(b) plots the corresponding phonon transmission functions. All the transmission spectra of these aperiodic structures are confined by those of the periodic and optimized aperiodic structures. The corresponding thermal conductivities at 100 and $300 \mathrm{~K}$ are shown in Fig. 7(c), where the black dashed lines are used as guides to recognize the level of the optimized aperiodic structure. The thermal conductivities of these aperiodic structures are higher than that of the optimized one but are lower than that of the periodic one. Therefore, the optimized aperiodic structure possesses the lowest conductivity, further validating the effectiveness of our MI-based optimization process. From the thermal conductivity values at 100 and $300 \mathrm{~K}$, the temperature only influences the absolute conductivity values, and has a negligible effect on the relative magnitudes. 


\section{CONCLUSIONS}

In summary, we demonstrate the ultimate controllability of coherent phonon thermal transport in the GaAs/AlAs SL structure using materials informatics. The optimal aperiodic SL that minimizes the coherent phonon heat conduction is designed by a method that alternatingly couples FP AGF and a Bayesian optimization. The designed optimal SL structure is fabricated by MBE. The thermal conductivity measurements over a wide temperature range from 77 to $300 \mathrm{~K}$ clearly demonstrate that the minimum thermal conductivity is experimentally realized. The excellent agreement between the fully coherent calculation and the experiments assures that the minimization of thermal conductivity is due to the optimal control of the coherent phonon thermal transport. Further, theoretical analysis identifies that the significant reduction in the optimal aperiodic SL from the finest periodic SL is attributed to the stronger and more extensive localization of the acoustic phonons. The optimal local structures that suppress phonon transmission at different frequency ranges are identified and validated to be mostly uncorrelated. The optimized aperiodic structure is formed by connecting these local structures with or without overlaps that introduce constructive or destructive interference over broad phonon frequencies. Such controllability and understanding of the aperiodic interference of coherent phonons opens a new route for phonon engineering to further suppress or enhance the thermal conductivities of various nanostructured materials.

\section{ACKNOWLEDGMENTS}

This work was partially supported by CREST "Scientific Innovation for Energy Harvesting Technology" (Grant No. JPMJCR16Q5), Grant-in-Aid for Scientific Research (A) (Grant No. 19H00744) from JSPS KAKENHI, and "Materials research by Information Integration" Initiative $\left(\mathrm{MI}^{2} \mathrm{I}\right)$ project from JST. R. H. would like to acknowledge the financial support by National Natural Science Foundation of China (Grant No. 51606074 and No. 51625601) and in particular, the JSPS Postdoctoral Fellowship (Grant No. P16369). K. H. acknowledges Grant-in-Aid from JSPS (Grants No. $15 \mathrm{H} 05868$ and No. 19K21957). The authors thank Dr. Takafumi Oyake for his support on the thermal measurements.

[1] G. Chen, Nanoscale Energy Transport and Conversion: A Parallel Treatment of Electrons, Molecules, Phonons, and Photons (Oxford University Press, Oxford, New York, 2005).

[2] M. Ohnishi and J. Shiomi, Towards Ultimate Impedance of Phonon Transport by Nanostructure Interface, APL Mater. 7, 013102 (2019).
[3] T. Oyake, L. Feng, T. Shiga, M. Isogawa, Y. Nakamura, and J. Shiomi, Ultimate Confinement of Phonon Propagation in Silicon Nanocrystalline Structure, Phys. Rev. Lett. 120, 045901 (2018).

[4] J. Maire, R. Anufriev, R. Yanagisawa, A. Ramiere, S. Volz, and M. Nomura, Heat Conduction Tuning by Wave Nature of Phonons, Sci. Adv. 3, e1700027 (2017).

[5] R. Anufriev, A. Ramiere, J. Maire, and M. Nomura, Heat Guiding and Focusing Using Ballistic Phonon Transport in Phononic Nanostructures, Nat. Commun. 8, 15505 (2017).

[6] J. Lee, W. Lee, G. Whlmeyer, S. Dhuey, D. L. Olynick, S. Cabrini, C. Dames, J. J. Urban, and P. D. Yang, Investigation of Phonon Coherence and Backscattering Using Silicon Nanomeshes, Nat. Commun. 8, 14054 (2017).

[7] A. Cepellotti, G. Fugallo, L. Paulatto, M. Lazzeri, F. Mauri, and N. Marzari, Phonon Hydrodynamics in Two-Dimensional Materials, Nat. Commun. 6, 6400 (2015).

[8] R. K. Wu, R. Hu, and X. B. Luo, First-Principle-Based Full-Dispersion Monte Carlo Simulations of the Anisotropic Phonon Transport in the Wurtzite GaN Thin Film, J. Appl. Phys. 119, 145706 (2016).

[9] T. Hori, J. Shiomi, and C. Dames, Effective Phonon Mean Free Path in Polycrystalline Nanostructures, Appl. Phys. Lett. 106, 171901 (2015).

[10] N. K. Ravichandran and A. J. Minnich, Coherent and Incoherent Thermal Transport in Nanomeshes, Phys. Rev. B 89, 205432 (2014).

[11] J. Garg, N. Bonini, B. Kozinsky, and N. Marzari, Role of Disorder and Anharmonicity in the Thermal Conductivity of Silicon-Germanium Alloys: A First Principle Study, Phys. Rev. Lett. 106, 045901 (2011).

[12] J. K. Yu, S. Mitrovic, D. Tham, J. Varghese, and J. R. Heath, Reduction of Thermal Conductivity in Phononic Nanomesh Structures, Nat. Nanotechnol. 5, 718 (2010).

[13] M. Maldovan, Phonon Wave Interference and Thermal Bandgap Materials, Nat. Mater. 14, 667 (2015).

[14] M. N. Luckyanova, J. Garg, K. Estarjani, A. Jandl, M. T. Bulsara, A. J. Schmidt, A. J. Minnich, S. Chen, M. S. Dresselhaus, Z.F. Ren, E. A. Fitzgerald, and G. Chen, Coherent Phonon Heat Conduction in Superlattices, Science 338, 936 (2012).

[15] J. Ravichandran, A. K. Yadav, R. Cheaito, P. B. Rossen, A. Soukiassian, S. J. Suresha, J. C. Duda, B. M. Foley, C. H. Lee, Y. Zhu, A. Lichtenberger, J. E. Moore, D. A. Muller, D. G. Schlom, P. E. Hopkins, A. Majumdar, R. Ramesh, and M. A. Zurbuchen, Crossover from Incoherent to Coherent Phonon Scattering in Epitaxial Oxide Superlattices, Nat. Mater. 13, 168 (2014).

[16] M. N. Luckyanova, J. Mendoza, H. Lu, B. Song, S. Huang, J. Zhou, M. Li, Y. Dong, H. Zhou, J. Garlow, L. Wu, B. J. Kirby, A. J. Grutter, A. A. Puretzky, Y. Zhu, M. S. Dresselhaus, and G. Chen, Phonon Localization in Heat Conduction, Sci. Adv. 4, eaat9460 (2018).

[17] Y. K. Koh, Y. Cao, D. G. Cahill, and D. Jena, HeatTransport Mechanisms in Superlattices, Adv. Funct. Mater. 19, 610 (2009).

[18] X. K. Chen, Z. X. Xie, W. X. Zhou, L. M. Tang, and K. Q. Chen, Phonon Wave Interference in Graphene and 
Boron Nitride Superlattice, Appl. Phys. Lett. 109, 023101 (2016).

[19] B. L. Davis and M. I. Hussein, Nanophotonic Metamaterials: Thermal Conductivity Reduction by Local Resonance, Phys. Rev. Lett. 112, 055505 (2014).

[20] L. Feng, T. Shiga, H. Han, S. Ju, Y. A. Kosevich, and J. Shiomi, Phonon Interference Resonance Effects by Nanoparticles Embedded in a Matrix, Phys. Rev. B 96, 220301 (R) (2017).

[21] R. Cheaito, C. A. Polanco, S. Addamane, J. Zhang, A. W. Ghosh, G. Balakrishnan, and P. E. Hopkins, Interplay Between Total Thickness and Period Thickness in the Phonon Thermal Conductivity of Superlattices from Nanoscale to the Microscale: Coherent versus Incoherent Phonon Transport, Phys. Rev. B 97, 085306 (2018).

[22] M. V. Simkin and G. D. Mahan, Minimum Thermal Conductivity of Superlattices, Phys. Rev. Lett. 84, 927 (2000).

[23] J. Garg and G. Chen, Minimum Thermal Conductivity in Superlattices: A First-Principle Formalism, Phys. Rev. B 87, 140302(R) (2013).

[24] B. Yang and G. Chen, Partially Coherent Phonon Heat Conduction in Superlattices, Phys. Rev. B 67, 195311 (2003).

[25] J. Mendoza and G. Chen, Anderson Localization of Thermal Phonons Leads to a Thermal Conductivity Maximum, Nano Lett. 16, 7616 (2016).

[26] S. Hu, Z. Zhang, P. Jiang, J. Chen, S. Volz, M. Nomura, and B. W. Li, Randomness-Induced Phonon Localization in Graphene Heat Conduction, J. Phys. Chem. Lett. 9, 3959 (2018).

[27] P. M. Norris, N. Q. Le, and C. H. Baker, Tuning Phonon Transport: From Interfaces to Nanostructures, J. Heat Transf. 135, 061604 (2013).

[28] R. Ramprasad, R. Batra, G. Pilania, A. M. Kanakkithodi, and C. Kim, Machine Learning in Material Informatics: Recent Applications and Prospects, npj Comput. Mater. 3, 54 (2017).

[29] G. J. Mulholland and S. P. Paradiso, Materials Informatics across the Product Lifecycle: Selection, Manufacturing, and Certification, APL Mater. 4, 053207 (2016).

[30] A. Agrawal and A. Choudhary, Material Informatics and Big Data: Realization of the Fourth Paradigm of Science in Materials Science, APL Mater. 4, 053208 (2016).

[31] A. Agrawal, P. D. Deshpande, A. Cecen, G. P. Basavarsu, A. N. Choudhary, and S. R. Kalidindi, Exploration of Data Science Techniques to Predict Fatigue Strength of Steel from Composition and Processing Parameters, Integr. Mater. Manuf. Innovation 3, 90 (2014).

[32] B. Meredig, A. Agrawal, S. Kirklin, J. E. Saal, J. W. Doak, A. Thompson, K. Zhang, A. Choudhary, and C. Wolverton, Combinatorial Screening for New Materials in
Unconstrained Composition Space with Machine Learning, Phys. Rev. B 89, 094104 (2014).

[33] R. Liu, A. Kumar, Z. Chen, A. Agrawal, V. Sundararaghavan, and A. Choudhary, A Predictive Machine Learning Approach for Microstructure Optimization and Materials Design, Sci. Rep. 5, 11551 (2015).

[34] P. V. Balachandran, J. Young, T. Lookman, and J. M. Rondinelli, Learning from Data to Design Functional Materials without Inversion Symmetry, Nat. Commun. 8, 14282 (2017).

[35] O. Isayev, C. Oses, C. Toher, E. Gossett, S. Curtarolo, and A. Tropsha, Universal Fragment Descriptors for Predicting Properties of Inorganic Crystals, Nat. Commun. 8, 15679 (2017).

[36] J. Carrete, W. Li, N. Mingo, S. Wang, and S. Curtarolo, Finding Unprecedentedly Low-Thermal-Conductivity HalfHeusler Semiconductors via High-Throughput Material Modeling, Phys. Rev. X 4, 011019 (2014).

[37] S. Ju, T. Shiga, L. Feng, Z. Hou, K. Tsuda, and J. Shiomi, Designing Nanostructures for Phonon Transport via Bayesian Optimization, Phys. Rev. X 7, 021024 (2017).

[38] M. Yamawaki, M. Ohnishi, S. Ju, and J. Shiomi, Multifunctional Structural Design of Graphene Thermoeletrics by Bayesian Optimization, Sci. Adv. 4, eaar4192 (2018).

[39] See Supplemental Material at http://link.aps.org/ supplemental/10.1103/PhysRevX.10.021050 for details of convergence tests, transmission function, preliminary study, experimental measurements, and additional analyses.

[40] T. Yamashita, N. Sato, H. Kino, T. Miyake, K. Tsuda, and T. Oguchi, Crystal Structure Prediction Accelerated by Bayesian Optimization, Phys. Rev. Mater. 2, 013903 (2018).

[41] A. J. Schmidt, X. Chen, and G. Chen, Pulse Accumulation, Radial Heat Conduction, and Anisotropic Thermal Conductivity in Pump-Probe Transient Thermoreflectance, Rev. Sci. Instrum. 79, 114902 (2008).

[42] N. C. Murphy, R. Wortis, and W. A. Atkinson, Generalized Inverse Participation Ratio as a Possible Measure of Localization for Interacting Systems, Phys. Rev. B 83, 184206 (2011).

[43] Y. Song, R. Wortis, and W. A. Atkinson, Dynamic Mean Field Study of the Two-Dimensional Disordered Hubbard Model, Phys. Rev. B 77, 054202 (2008).

[44] G. Schubert, J. Schleede, K. Byczuk, H. Fehske, and D. Vollhardt, Distribution of the Local Density of States as a Criterion for Anderson Localization: Numerically Exact Results for Various Lattices in Two and Three Dimensions, Phys. Rev. B 81, 155106 (2010).

[45] T. Yamamoto, K. Sasaoka, and S. Watanabe, Universality and Diversity in a Phonon-Transmission Histogram of Isotope-Disordered Carbon Nanotubes, Phys. Rev. Lett. 106, 215503 (2011). 